\title{
An Energy Balanced Reliable Routing Metric in WSNs
}

\author{
Jian ZHU, Hai ZHAO, Jiuqiang XU \\ The Northeastern University, Shen Yang, China \\ E-mail: zhujian1981710@163.com \\ Received November 13, 2008; revised February 16, 2009; accepted February 18, 2009
}

\begin{abstract}
In WSNs' applications, not only the reliable end-to-end communications are must be ensured, but also the reduction of energy consumption and the entire network's lifetim e should be optimized. All of the above have become to be an important way to evaluate the performance of routing protocols. In this paper, an optimization model for WSNs' lifetime is firstly advanced. Secondly, the shortage of ETX based routing metric is solved with the help of the optimization model. Thirdly, an energy balanced routing metric is advanced which is called EBRM in this paper. The result of simulation in NS-2 shows that, the EBRM metric can not only prolong the network's lifetime, but also can ensure the reliable end-to-end communication.
\end{abstract}

Keywords: WSN, Routing Metric, Reliable Communication, Lifetime, Energy Balance

\section{Introduction}

The WSNs (Wireless Sensor Networks) is a kind of selforganized network which mainly aims at applications. The wireless links are not stable, and the loss of packets happens frequently in communications [1,2]. To solve this problem, some routing metrics are adopted in routing protocol, for example, the ETX (expected transmission) metric can make a most reliable link to be used. But, the WSNs is mainly battery-powered, the energy is restricted, whether the sensor or monitor applications often need a network with more than one year lifetime. If we only consider the reliability of communication, some of the WSNs nodes will be exhausted rapidly. Unfortunately, the few exhausted nodes are always very important to the entire network, if they can't work, the network's performance will be pulled down and the entire network's lifetime will be reduced. So, how to balance the load on the base of reliable communication become to be a key problem in WSNs $[3,4]$.

Aiming at the problems above, an energy balanced reliable routing metric is advanced-EBRM (Energy Balanced Reliable Metric). This metric can not only ensure the reliable communication, but also balance the network's load, and prolong the networks' lifetime.

\section{Mode of Lifetime's Optimization}

Before optimizing the lifetime of network, we must know that, how to measure the lifetime. In different applications, the common ways are as follow: the ratio of remained active nodes in network [5], packets reception ratio [6] and the time of first blind covering's appearance [7]. The mutual character of above ways is that, while the index of network reaches a set value, the network's lifetime is considered to be over. But, in different applications, it is very difficult to make out a universal index value to judge the network's life.

So, in this paper, we use the time of the network node's first death as the measurement of network's lifetime [8]. This metric's usage range is wider, and it is used more early, it supposes that, all the nodes place the same part in the network. Its excellence is that, it reflects the hypostasis of the lifetime maximization problem, and this hypostasis is network's load balance.

Thus, we can set up the model of network's lifetime. It is supposed that, there are $\mathrm{S}$ sensor nodes in a network, every node sends a packet an second, and every node $s$ has $D_{s}$ paths to destination node $r$. Make $h_{s, p, r}$ as the hops between node $s$ and node $r$ in path $p$, if node $r$ doesn't belong to path $p$, the $h_{s, p, r}$ is set to be $+\infty$. So, the probability of relaying the packet which comes from node $s$ is $s_{s, p, r}^{t x}$, its expression is as follow:

$$
\left\{\begin{array}{cl}
\prod_{k \in K_{s, p, r}} P_{s}\left(G_{s, p, k}, l_{s, p, k}\right), & \text { if } 0<h_{s, p, r}<\infty \\
1, & \text { if } h_{s, p, r}=0 \\
0, & \text { if } h_{s, p, r}=\infty
\end{array}\right.
$$

In the above expression:

$$
K_{s, p, r} \equiv\left\{k \mid 0 \leq h_{s, p, k}<h_{s, p, r}\right\}, G_{s, p, k} \text { is the channel gain }
$$
of correspond links, $l_{s, p, k}$ is the sending power level of 
node $k$. The probability of the packets which come from node $\mathrm{s}$ received by node $r$ is $s_{s, p, r}^{r x}$, and it equals to the probability of the packets sent by node $r$ 's upstream node, it is node $\mathrm{k}$ and $h_{s, p, k}=h_{s, p, r-1}$. So,

$$
s_{s, p, r}^{r x}=s_{s, p, k}^{t x}
$$

Therefore, if sensor node $i$ want to send some packets, the energy's decrease speed of node $i$ is $V_{E}$ :

$$
\sum_{s=1}^{S} \sum_{p=1}^{D s} A\left(s_{s, p, i}^{t x} E_{s, p, i}^{t x}+s_{s, p, i}^{r x} E_{s, p, i}^{r x}\right) u_{s, p}
$$

And

$$
\begin{gathered}
E_{s, p, i}^{t x}=E^{t x}\left(P_{l_{s, p, i}}\right)+E^{a c k-r x} \\
E_{s, p, i}^{r x}=E^{r x}+E^{a c k-t x}
\end{gathered}
$$

The $u_{s, p}$ is ratio of path $p$ 's holding time which is used by node $s$. So, node $i$ 's lifetime can be expressed by: $T_{i}=\frac{E_{i}}{V_{E}}, E_{i}$ is the initialization energy of node $i$.

$$
\begin{aligned}
V_{E}= & \sum_{s=1}^{S} \sum_{p=1}^{D s} A\left(E_{s, p, i}^{t x} N_{s, p, i}^{t x}+E_{s, p, i}^{r x} N_{s, p, i}^{r x}\right. \\
& \left.+E_{s, p, i}^{a c k-t x} N_{s, p, i}^{a c k-t x}+E_{s, p, i}^{a c k-r x} N_{s, p, i}^{a c k-r x}\right) u_{s, p}
\end{aligned}
$$

According to the above metric, the WSNs' lifetime can be defined as $T=\min _{i} T_{i}$.

So, the problem of WSNs' lifetime can be transformed to be a linear programming problem: Minimizing 1/T.

$$
\begin{gathered}
\frac{1}{T}=\frac{A}{E_{i}} \sum_{s=1}^{S} \sum_{p=1}^{D s}\left(s_{s, p, i}^{t x} E_{s, p, i}^{t x}+s_{s, p, i}^{r x} E_{s, p, i}^{r x}\right) u_{s, p} \\
\sum_{p=1}^{D s} u_{s, p}=1, \text { for all } \mathrm{s} \\
u_{s, p} \geq 0, \text { for all } \mathrm{s}, \mathrm{p}
\end{gathered}
$$

The expression (7) indicates the speed of node's energy consumption, it is easy to find that, if we decrease the power consumption in network's communication, the longer lifetime of network can be got, the correlation researches mostly focus on physical layer and link layer. From the expression (8), we can got that, the node $i$ is used in $D_{s}$ paths and following a specifically time ratio $u_{s, p}$, for example, if the ratio $u_{s, q}$ equals to zero, it is said that, the node $i$ will not be used in path $q$. So, we can adjust the value of $u_{s, p}$ to solve the above linear programming problem. In this paper, we increase the network's lifetime mainly by balancing the network's load.

\section{Energy Balanced Reliable Routing Metric}

Because of the complexity and un-reliability in wireless communications, there are many differences between wired network and wireless network, so, their routing protocols are also different. The routing metrics place a more and more important part in routing protocols' design and performance; every routing metric indicates the cost of path selection and routing capability.

While in routing finding or routing table's maintenance, the value of routing metric should firstly be got from routing table, and then decide the next hop according to the metric values. ETX is a metric which aims at high reliable communication [9], and the value equals to the final logic transmission times while one packet wants to be successfully transmitted, it includes retransmissions, the value $\mathrm{ETX}=1 / p q$ ( $p$ is the forward packet reception ratio, $q$ is the reverse packet reception ratio). The ETX value of a path equals to the summation of every hop's ETX in the path.

The ETX mainly aims at reliable communication, however, if only the ETX is used in WSNs, it maybe cause a too many hops path, and the high quality paths are frequently used. So the lifetime of the high quality paths will be decreased, thereby, the entire network's lifetime and quality will be decreased. On the basis of ETX, a reliable and network load balanced routing metric-EBRM is advanced.

The main idea of EBRM is that, the time ratio of a node is according to the node's remainder energy, for example, the initialization energy is set to be $\mathrm{E}$, and the energy has $\mathrm{N}$ levels, in several reachable paths, the $\mathrm{M}$ paths with the highest energy level are firstly selected, and secondly, the most reliable path in the selected $\mathrm{M}$ path is finally selected. The routing method can not only ensure the end-to-end reliability, but also can decrease the load of the nodes whose energy is in lowest level. So the load of network is optimized, and the lifetime of the network can be prolonged.

The EBRM is not a routing protocol, it is a metric, so, it can only be used in a routing protocol, it is said that, the existed protocol's metric will be changed by EBRM. The AODV [10] is a very classical protocol; it is very suitable for WSNs' applications. The most important is that, the AODV uses hello packets to maintenance the routing table, the hello packets are useful for EBRM. The metric of AODV is Hops; it selects the path which has the least Hops. Because of the character of wireless channel, the Hops-AODV can't be used in WSNs. So the AODV is improved in this paper, it is called EBRMAODV; the main processes are as follow:

1) In the network initialization, every nodes send fifty packets to each other, and compute the forward and reverse packet reception ratio $p$ and $q$, so, the ETX between two neighbor nodes can be got; In this paper, the remainder energy of a node is defined to be four level

$\{$ Elevel | Elevel=1,2,3,4 $\}$, actually the number of energy level can equal to be any natural numbers according to the requirements of user. A small number of energy levels will cause a low performance in energy balance, but a high performance in reliable communication, for example, if the number of energy level is set to be one, it means that, all the paths will always have the same energy level, and the most reliable path will be in used until 
it is dead; Accordingly, a big number of energy level will cause a high performance in energy balance, but a low performance in reliable communication. In our experiment, we define the energy to be four levels subjectively.

2) The source node $s$ broadcasts a routing request packet (RREQ). The RREQ of AODV is advanced as Figure 1, the original metric-Hops is replaced by ETX and minElevel, the value of ETX in Figure 1 is ETX of a path, it is an accumulative total, for example, if there are three hops between two nodes, every hop has a ETX, and the path's ETX equals to the summation of the three hop ETXs; The value of minElevel is the lowest energy level of all the nodes in a candidate path.

3) The relay node $M_{i}$ which receives the RREQ adds the ETX $_{\mathrm{i}, \mathrm{i}-1}$ (the ETX between node $\mathrm{M}_{\mathrm{i}}$ and node $\mathrm{M}_{\mathrm{i}-1}$ ) and the current ETX value in Figure 1. So, while the RREQ reaches to a destination node, the ETX in Figure 1 equal the path's ETX.

4) At the time of sending packets, source node $s$ will firstly broadcast RREQ to find out the reachable paths $P_{i}$ to destination node $d$, after the RREQ reaches in $d$, a packet called RREP will be send back to $s$ in paths $P_{i}$. In the broadcasting, every relay node which receives the

\begin{tabular}{|c|c|c|c|c|}
\hline type & symbol & Reserved & ETX & $\min E_{\text {level }}$ \\
\hline \multicolumn{5}{|c|}{ RREQ identifier } \\
\hline \multicolumn{5}{|c|}{ Destination IP } \\
\hline \multicolumn{5}{|c|}{ Destination sequence } \\
\hline \multicolumn{5}{|c|}{ Source IP } \\
\hline \multicolumn{5}{|c|}{ Source sequence } \\
\hline
\end{tabular}

Figure 1. The advanced format of RREQ.
RREQ will count its energy level, if it is less than the minElevel which is recorded in received RREQ, the value of minElevel in RREQ packet will be updated to be the node's lower energy level.

5) After receiving the RREQ, the destination node will firstly select several paths with highest energy level. In these paths, the most reliable path can be got by the use of ETX.

6) The RREQ is sent periodically by source node to update the routing table, and check the power levels' change of the nodes. Along with the usage of nodes, if a currently used path's minElevel is lower than a candidate path's, then, the candidate path will be used. By this, the network's load can be balanced, and the energy cost is also balanced.

The flow figure of EBRM-AODV is as follow:

So, while the EBRM is used in a network, the recorded information will be updated frequently, the path which has a bigger minElevel will be selected. This means that, if a path's energy is decreased, the EBRM will select another path which has more energy. So, every path's lifetime is prolonged, and the quality of the network is advanced.

\section{Simulation of EBRM Metric}

The EBRM's capability is analyzed by the result of simulation in NS-2. The scene is as follow: there are eighty nodes in a $100 \mathrm{~m} \times 100 \mathrm{~m}$ square area, the detailed parameters of nodes are as Table 1:

The routing protocol is AODV whose routing metric is Hops, ETX or EBRM; the three metrics' capability will be compared. In the simulations, the Energy Model is used, and the parameters of a node are set to be as follow: the initial energy equals to 1 , and every packet's sending costs 0.002 , every hello packet's cost 0.001 and every packet's receiving costs 0.0005 .

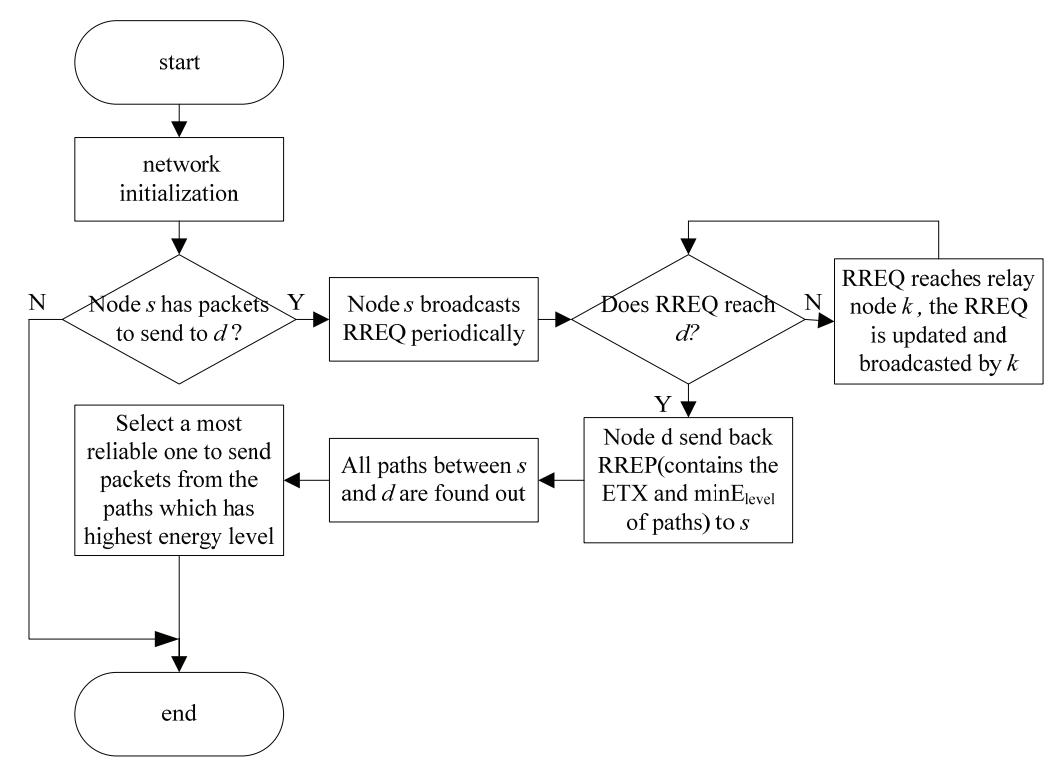

Figure 2. The flow figure of EBRM-AODV. 
Table 1. The detailed parameters of nodes in NS-2.

\begin{tabular}{lc}
\hline \multicolumn{1}{c}{ Parameter } & Value \\
\hline Nodes distribution & Random \\
Channel bandwidth & $250 \mathrm{kbps}$ \\
Frequency & $2.4 \mathrm{GHz}$ \\
Wireless model & Shadowing \\
MAC protocol & IEEE 802.15 .4 \\
$\begin{array}{l}\text { Power for transmission } \\
\text { (mWatt) }\end{array}$ & 2.0 \\
$\begin{array}{l}\text { Power for idle (mwatt) } \\
\text { Power for reception(mwatt) }\end{array}$ & 1.0 \\
$\begin{array}{l}\text { Power for sleep (mwatt) } \\
\text { Power for sleep/idle transi- } \\
\text { tion (mWatt) }\end{array}$ & 1.0 \\
$\begin{array}{l}\text { Time for sleep/idle transi- } \\
\text { tion (s) }\end{array}$ & 0.001 \\
\hline
\end{tabular}

Aiming at the three metrics' capabilities, two experimentations are designed. The first experiment is that, the $p l r$ (packet lost ratio) and the remainder energy are tested without packet retransmissions; the second experiment is that, the network's lifetime with different reliability requirements is tested with packet retransmissions.

In the first experiment, the plr's calculation is

$$
P_{\text {lost }}=\frac{N_{\text {source }}-\mathrm{N}_{\text {destination }}}{N_{\text {source }}}
$$

The $\mathrm{N}_{\text {source }}$ is a number of the packets which are sent by source node, $\mathrm{N}_{\text {destimation }}$ is a number of the packets which are received by destination node, and the result is as Figure 3.

It shows that, the routing's set up is almost finished after thirty seconds, the plr of ETX-AODV is lowest, this is because that, the ETX metric mainly aims at reliable communications, however, the Hops metric aims at shortest path, it doesn't consider the reliability, so, Hops-AODV causes a highest plr. The EBRM metric gives attention to energy balance, so, its reliability is a little bigger than Hops' and smaller than ETX's.

In the same setting, the relationship between the average remainder energy of all nodes in entire network and time is shown in Figure 4.

Figure 4 shows that, the EBRM metric can cause lower energy consumption than ETX metric, and cause higher energy consumption than Hops metric. This is because that, Hops-AODV aims at shortest path, and shortest path can decrease the number of working nodes, so the shorter path will cause lower energy consumption.

The ETX metric will not select the shortest path to transmit packets. For a low plr, it only selects the path with highest reliability, so it causes lower average remainder energy than EBRM.

In the second experiment, a retransmission mechanism is added into IEEE802.15.4 to ensure the reliability communication. With the increase of reliability, the three metrics based on network's lifetime are shown in Figure 5.

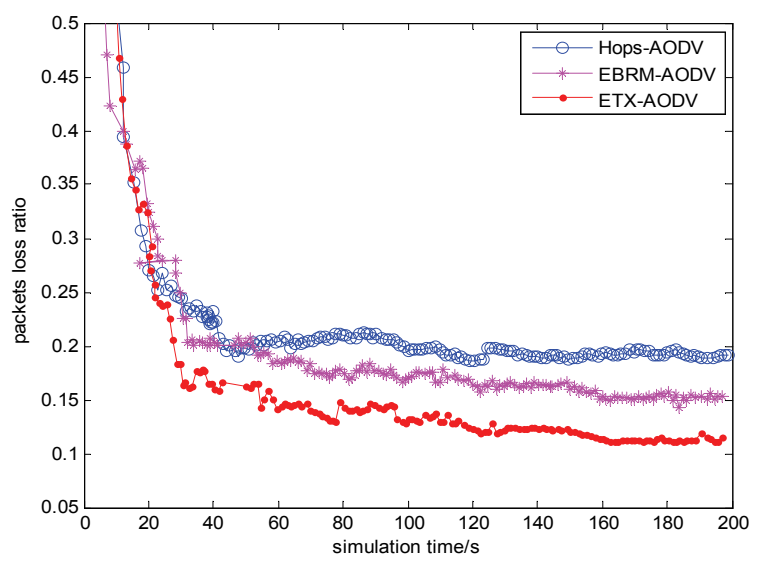

Figure 3. The relationship between plr and time.

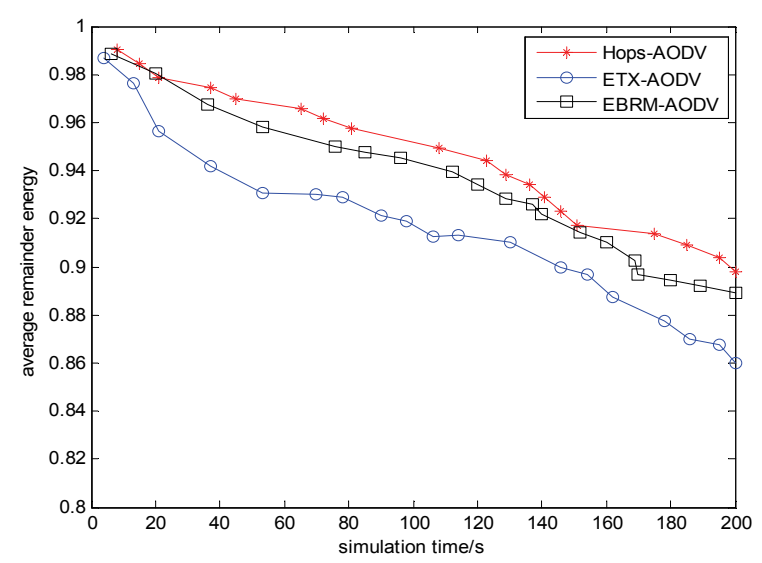

Figure 4. The relationship between remainder energy and time.

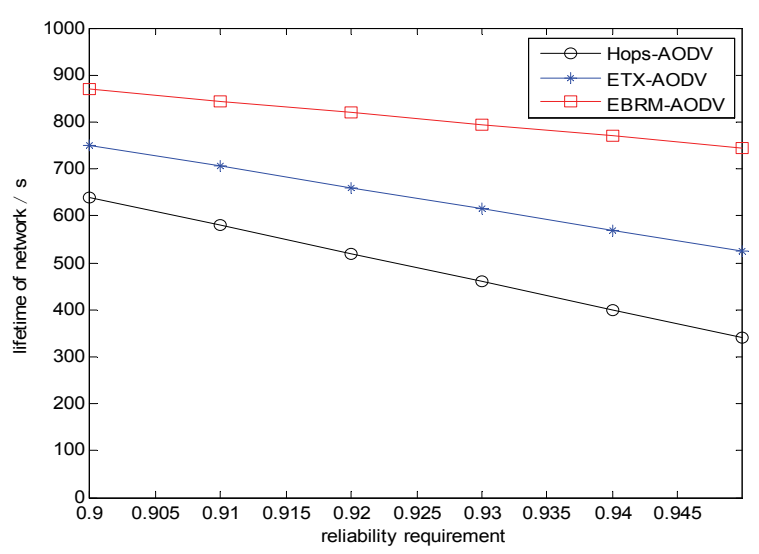

Figure 5. Lifetime with different reliability requirements.

From Figure 5, the EBRM metric can cause a longest lifetime, and with the reliability's increase, the three lifetimes are all decreased. The lifetime with Hops metric drops fastest, it is because that, Hops based protocol will cause a large number of retransmission packets, it will not only consume a mass of energy, but also can make some important nodes dead quickly. The ETX metric considers the reliability, and the number of retransmission packets is decreased, so it will decrease the energy 


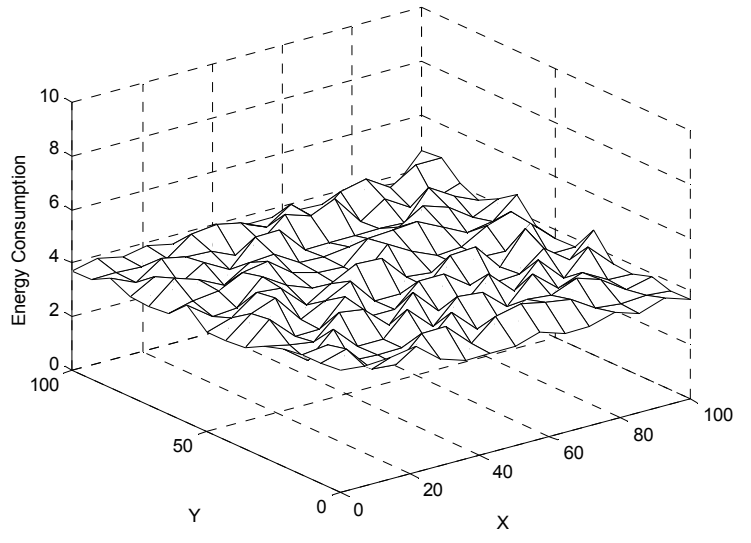

Figure 6. Energy consumption in energy balanced network.

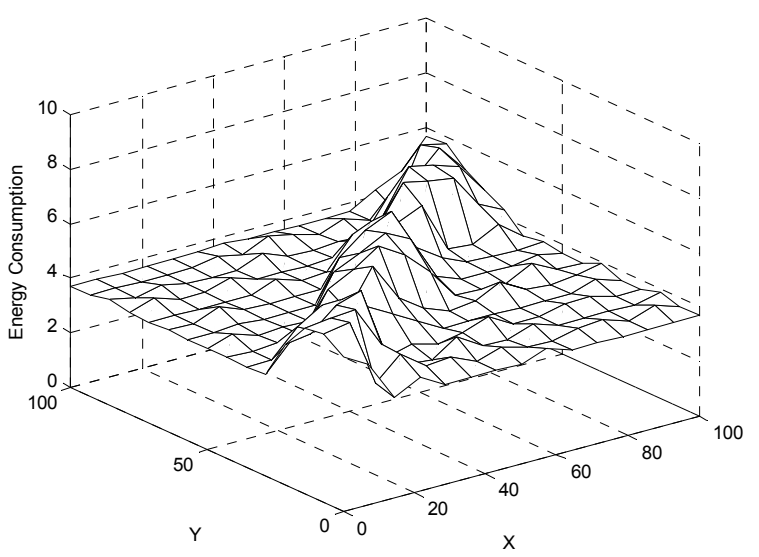

Figure 7. Energy consumption in no energy balance network.

consumption. But, ETX doesn't consider the entire network's energy balance; some important nodes will die quickly as the Hops metric.

To describe the energy balance lively, the result of energy balanced network and no energy balance network in a same setting are shown in Figure 6 and Figure 7.

In Figure 6 and Figure 7, axis $\mathrm{X}$ and axis $\mathrm{Y}$ construct a square area where the nodes are distributed. The two figures show that, the energy consumption in Figure 7 is very unbalanced, some nodes are consumed heavily, but, the other nodes are consumed lightly. While the heavily consumed nodes dead, the entire network is almost dead, so, the lifetime of the network is decreased heavily. In Figure 6 , the problem of energy balance is solved, the network's load is balanced in the all nodes, every node's energy is almost exhausted, this can prolong the network's lifetime as long as possible. So the EBRM metric is advanced, it can balance the energy consumption and ensure the reliability, the entire network's lifetime is prolonged.

\section{Conclusions}

This paper optimizes the WSNs' reliability and lifetime, and advances a routing metric called EBRM. From the result of simulation, the metric can balance the entire network's lifetime by balancing the energy consumption on the base of reliable communication, this metric is more suitable for low reliability and energy restricted WSNs' applications.

\section{References}

[1] I. F. Akyildiz, W. Su, Y. Sankarasubramaniam, et al., "A survey on sensor networks," [J]. IEEE Communications Magazine, 40(8), pp. 102-105, 2002.

[2] C. Suh, Y. B. Ko, D. M. Son, et al., "Energy efficient cross-layer MAC protocol for wireless sensor networks," [A]. Proceedings of the APWeb 2006 International Workshops: XRA, IWSN, MEGA, and ICSE[C]., Harbin, Springer Verlag, pp. 410-419, 2006.

[3] H. L. Van, T. Nieberg, J. Wu, et al., "Prolonging the lifetime of wireless sensor networks by cross-layer interaction," [J]. IEEE Wireless Communications, 11(6), pp. 78-86, 2004.

[4] I. Y. Kong and W. J. Hwang, "Lifetime maximization by cross-layer interaction in wireless sensor networks," [A]. Proceedings of the 8th International Conference Advanced Communication Technology (ICACT 2006) [C]. Phoenix Park: Institute of Electrical and Electronics Engineers Computer Society, pp. 2055-2060, 2006.

[5] P. J. Wan, G. Calinescu, and X. Li, "Minimum-energy broadcast routing in static ad-hoc wireless networks," [C]. Proceedings of INFOCOM'01, Anchorage, AK, pp. 1162-1171, 2001.

[6] C. K. Toh, "Maximum battery life routing to support ubiquitous mobile computing in wireless ad hoc networks," [J]. IEEE Communications Magazine, pp. 138-147, 2001.

[7] M. Bhardwaj, T. Garnett, A. P. Chandrakasan, "Upper bounds on the lifetime of sensor networks," [C]. Proceedings of ICC, Helsinki, Finland, pp. 785-790, 2001.

[8] S. Singh, M. Woo, and C. S. Raghavendra, "Power-aware routing in mobile ad hoc networks," [C]. Proceedings of the 4th Annual ACM/IEEE International Conference on Mobile Computing and Networking, Dallas, United States, ACM Press, 1998.

[9] D. Couto, "High-throughput routing for multi-hop wireless networks," [D]. Massachusetts, Massachusetts Institute of Technology, pp. 55-60, 2004.

[10] C. Perkins and E. Royer, "Ad hoc on-demand distance vector routing," Proceedings of the 2nd IEEE Workshop on Mobile Computing Systems and Applications, 1999. 